\title{
Measurement of the neutron electric form factor via recoil polarimetry
}

\author{
The Jefferson Laboratory E93-038 Collaboration
}

T. Reichelt ${ }^{16, a}$, R. Madey ${ }^{1,2}$, A.Yu. Semenov ${ }^{1}$, S. Taylor ${ }^{2}$, A. Aghalaryan ${ }^{4}$, E. Crouse $^{5}$, G. MacLachlan $^{6}$, B. Plaster $^{3}$, S. Tajima ${ }^{7}$, W. Tireman ${ }^{1}$, Chenyu Yan ${ }^{1}$, A. Ahmidouch ${ }^{8}$, B.D. Anderson ${ }^{1}$, R. Asaturyan ${ }^{4}$, O. Baker ${ }^{9}$, A.R. Baldwin ${ }^{1}$, H. Breuer ${ }^{10}$, R. Carlini ${ }^{2}$, E. Christy ${ }^{9}$, S. Churchwell ${ }^{7}$, L. Cole ${ }^{9}$, S. Danagoulian ${ }^{2,8}$, D. Day ${ }^{11}$, M. Elaasar ${ }^{12}$, R. Ent ${ }^{2}$, M. Farkondeh ${ }^{3}$, H. Fenker ${ }^{3}$, F.M. Finn ${ }^{5}$, L. Gan ${ }^{9}$, K. Garrow ${ }^{2}$, P. Gueye ${ }^{9}$, C. Howell ${ }^{7}$, B. Hu ${ }^{9}$, M.K. Jones ${ }^{2}$, J.J. Kelly ${ }^{10}$, C. Keppel ${ }^{9}$, M. Khandaker ${ }^{13}$, W.Y. Kim ${ }^{14}$, S. Kowalski ${ }^{3}$, A. Lung ${ }^{3}$, D. Mack ${ }^{2}$, D.M. Manley ${ }^{1}$, P. Markowitz ${ }^{15}$, J. Mitchell ${ }^{2}$, H. Mkrtchyan ${ }^{4}$, A.K. Opper 6 , C. Perdrisat ${ }^{5}$, V. Punjabi ${ }^{13}$, B. Raue ${ }^{15}$, J. Reinhold ${ }^{15}$, J. Roche ${ }^{5}$, Y. Sato ${ }^{9}$, W. Seo ${ }^{14}$, N. Simicevic ${ }^{17}$, G. Smith ${ }^{2}$, S. Stepanyan ${ }^{4}$, V. Tadevosyan ${ }^{4}$, L. Tang ${ }^{9}$, P. Ulmer ${ }^{18}$, W. Vulcan ${ }^{2}$, J.W. Watson ${ }^{1}$, S. Wells ${ }^{17}$, F. Wesselmann ${ }^{11}$, S. Wood ${ }^{2}$, Chen Yan ${ }^{2}$, L. Yuan ${ }^{9}$, S. Yang ${ }^{14}$, W.M. Zhang ${ }^{1}$, H. Zhu ${ }^{11}, \mathrm{X} . \mathrm{Zhu}^{9}$, and H. Arenhövel ${ }^{19}$

1 Kent State University, Kent, OH 44242, USA

2 Thomas Jefferson National Accelerator Facility, Newport News, VA 23606, USA

3 Massachusetts Institute of Technology, Cambridge, MA 02139, USA

4 Yerevan Physics Institute, Yerevan 375036, Armenia

5 The College of William and Mary, Williamsburg, VA 23187, USA

6 Ohio University Athens, $\mathrm{OH}$ 45701, USA

7 Duke University, Durham, NC 27708, USA

8 North Carolina A \& T State University, Greensboro, NC 27411, USA

9 Hampton University, Hampton, VA 23668, USA

10 University of Maryland, College Park, MD 20742, USA

11 University of Virginia, Charlottesville, VA 22904, USA

12 Southern University of New Orleans, New Orleans, LA 70126, USA

13 Norfolk State University, Norfolk, VA 23504, USA

14 Kyungpook National University, Korea

15 Florida International University, Miami, FL 33199, USA

16 Rheinische Friedrich-Wilhelms-Universität Bonn, Germany

17 Louisiana Tech University, Ruston, LA 71272, USA

18 Old Dominion University, Norfolk, VA 23508, USA

19 Johannes Guthenberg-Universität Mainz, Germany

Received: 30 September 2002 /

Published online: 22 October 2003 - (c) Società Italiana di Fisica / Springer-Verlag 2003

\begin{abstract}
The ratio $G_{e}^{n} / G_{m}^{n}$ of the electric to the magnetic form factor of the neutron has been measured by analyzing the polarization of the recoiling neutron in quasi-elastic scattering of longitudinally polarized electrons from deuterium at the $Q^{2}$ values of $0.45,1.15$, and $1.47(\mathrm{GeV} / c)^{2}$. The experiment has been performed in Hall C of the Thomas Jefferson National Accelerator Facility. With $G_{m}^{n}$ being known $G_{e}^{n}$ can be deduced. The preliminary results show that the lowest $Q^{2}$ points follow the Galster parametrization and that the $1.47(\mathrm{GeV} / c)^{2}$ point rises above this parametrization.
\end{abstract}

PACS. 13.40.Gp Electromagnetic form factors - 14.20.Dh Protons and neutrons - 24.70.+s Polarization phenomena in reactions

\section{Introduction}

The electromagnetic form factors of the nucleon are fundamental quantities in hadron pysics. In the past the lack of

\footnotetext{
a e-mail: reichelt@physik.uni-bonn.de
}

data was unsatisfactorily severe for $G_{e}^{n}$, the electric form factor of the neutron. In the last years, however, substantial progress has been made by developing high-quality polarized electron beams and efficient neutron polarimeters allowing the measurement of the interference term in 


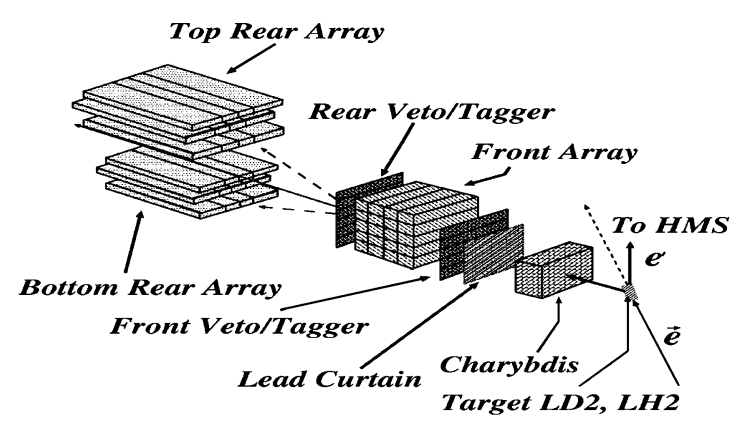

Fig. 1. Experimental set-up.

polarization experiments and extracting the electric form factor with small systematic and statistical errors.

\section{Experimental method}

A sketch of the experimental set-up is shown in fig. 1 . A beam of longitudinally polarized electrons scattered from a $15 \mathrm{~cm}$ liquid deuterium target. The scattered electrons were detected and momentum analyzed in the HighMomentum Spectrometer (HMS) of Hall C in coincidence with the recoiling neutrons, the polarization of which was measured in the polarimeter NPOL. NPOL consisted of an array of front detectors (20) and two arrays of rear detectors (12 each). It made use of the spin-dependent scattering of the neutrons in the front detectors resulting in an up/down asymmetry which is proportional to the transverse component of the polarization vector.

The $100 \mathrm{~cm} \times 10 \mathrm{~cm} \times 10 \mathrm{~cm}$ dimensions of each front detector were small enough to permit high luminosity. A double layer of thin tagging detectors directly ahead of and behind the front array identified charged particles. Each layer of the rear array consisted of two central scintillators, each $25.4 \mathrm{~cm} \times 10.16 \mathrm{~cm} \times 101.6 \mathrm{~cm}$, with a $50.8 \mathrm{~cm} \times 10.16 \mathrm{~cm} \times 101.6 \mathrm{~cm}$ scintillator on each side. A $10 \mathrm{~cm} \mathrm{~Pb}$ curtain attenuated the flux of electromagnetic background from the target. The two sides of NPOL were shielded by $\sim 1.2 \mathrm{~m}$ concrete, the rear and front shielding consisted of steel blocks of $\sim 1.3 \mathrm{~m}$ thickness. A collimator in the front shielding prevented the rear detectors from direct sight to the target. The mean flight path for the neutrons was $7 \mathrm{~m}$. Rejection of inelastic events was accomplished with two cuts: a missing-momentum cut of less than $100 \mathrm{MeV} / c$, and a relative momentum bite $\mathrm{d} P / P$ of $-3 \%$ to $+5 \%$. The resulting solid angle for NPOL was 10 msr. Throughout the experiment NPOL remained positioned at 46 degrees relative to the beam. For the different $Q^{2}$ values the beam energy and the HMS angle and momentum were adjusted accordingly.

The polarization vector of the neutrons leaving the target lies in the scattering plane. The longitudinal component is given by kinematics when $G_{e}^{n}$ is negligible. The transverse component is proportional to the ratio $G_{e}^{n} / G_{m}^{n}$. The dipole magnet Charybdis was positioned between the target and NPOL and the field was vertical. The interaction of the neutron's magnetic moment with the field

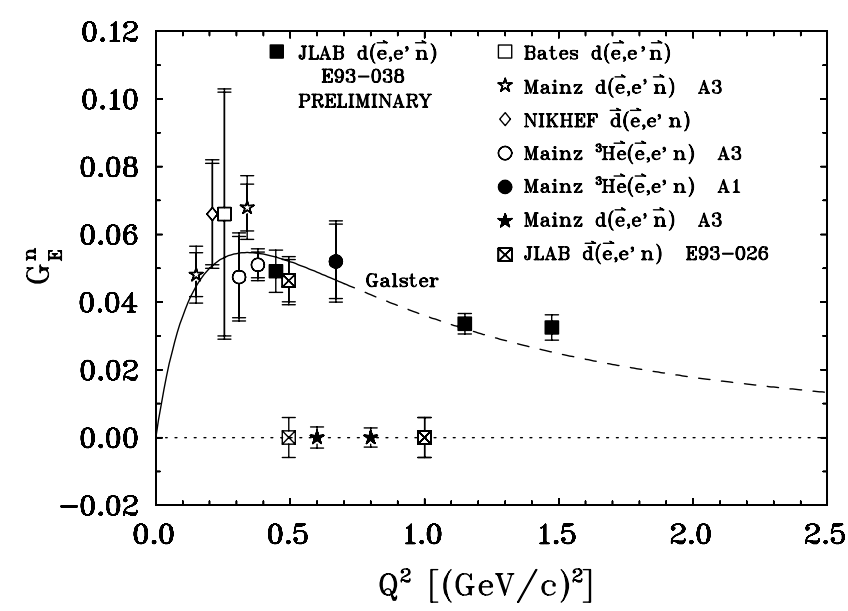

Fig. 2. World data on $G_{e}^{n}$ vs. $Q^{2}$ as obtained from polarization experiments. The points on the abscissa are projections.

resulted in a precession of the polarization vector by an angle $\chi$.

When a pair of asymmetry measurements with two different precession angles is taken, the ratio $g=G_{e}^{n} / G_{m}^{n}$ can directly be expressed as a function of the precession angles $\chi$ and the ratio $\eta$ of the corresponding asymmetries $\xi$. We chose the precession angles

$$
\begin{array}{lll}
\chi=0^{\circ} / 90^{\circ}, & \eta=\xi_{0} / \xi_{90}, & g=-K \cdot \eta \quad \text { and } \\
\chi=-40^{\circ} /+40^{\circ}, & \eta=\xi_{-} / \xi_{+}, & g=-K \cdot \tan \chi \cdot \frac{\eta+1}{\eta-1},
\end{array}
$$

where $K$ is a known kinematical factor. A remarkable advantage of the ratio technique lies in the fact that in the ratio of the asymmetries the analyzing power and also the beam polarization cancel, provided the latter is stable.This has been verified by repeated measurements with the $\mathrm{C}$ beam-line Møller polarimeter.

\section{Scattering asymmetries}

An event is generated by a triple coincidence between an electron in the HMS, a neutral particle in the front array and either a neutral or charged particle in one of the rear arrays. TOF spectra were recorded for the time of flight between the HMS and a front detector (ctof) and between the front detector and a rear detector (dtof). These spectra allowed the subtraction of accidentals. The four dtof spectra (up, down, + helicity, - helicity) contain the information from which the asymmetry is extracted. From the yields in the dtof peaks, the ratio $r$ is calculated, which is the ratio of the two geometric means $\left(N_{U}^{+} N_{D}^{-}\right)^{1 / 2}$ and $\left(N_{U}^{-} N_{D}^{+}\right)^{1 / 2}$, where $N_{U}^{ \pm}, N_{D}^{ \pm}$are the number of events for nucleons scattered up (down) when the beam helicity was positive (negative). The physical asymmetry is then given by $(r-1) /(r+1)$. By this method inherent asymmetries like different solid angles for the rear arrays or a beam charge asymmetry for the two helicity states cancel. 


\section{Preliminary results}

In fig. 2 our preliminary results are plotted vs. $Q^{2}$ together with the current world data for $G_{e}^{n}$ from polarization experiments [1-9]. The data from E 93-038 follow the Galster parametrization [10] up to $1.15(\mathrm{GeV} / c)^{2}$ and rises above at $1.47(\mathrm{GeV} / c)^{2}$. Schiavilla and Sick [11] extracted values for $G_{e}^{n}$ from the nuclear physics data on the quadrupole form factor of the deuteron and obtained results up to $Q^{2} \sim 1.65(\mathrm{GeV} / c)^{2}$ consistent with the Galster parametrization. Our data together with those to be expected in the near future (Mainz, JLab) constitute a challenge for theoretical nucleon models [12-19], which must be able to reproduce all four electromagnetic form factors of the nucleon. They also allow a better determination of the interior charge distribution of the neutron [20].

The support of the Jefferson Lab scientific and engineering staff in Hall $\mathrm{C}$ and the accelerator staff is gratefully acknowleged. This work was supported in part by the National Science Foundation, the Department of Energy, and the Deutsche Forschungsgemeinschaft. The Southeastern Universities Research Association (SURA) operates the Thomas Jefferson National Accelerator Facility under the U.S. Department of Energy contract DE-AC05-84ER40150.

\section{References}

1. T. Eden et al., Phys. Rev. C 50, R 1749 (1994).

2. M. Meyerhoff et al., Phys. Lett. B 327, ( 1994), 201

3. J. Becker et al., Eur. Phys. J A 6, 329 (1999).

4. J. Golak et al., Phys. Rev. C 63, 034006-1 (2001).

5. M. Ostrick et al., Phys. Rev. Lett. 83, 276 (1999).

6. C. Herberg et al., Eur. Phys. J. A 5, 131 (1999).

7. I. Passchier et al., Phys. Rev. Lett. 82, 4988 (1999).

8. D. Rohe et al., Phys. Rev. Lett. 83, 4257 (1999).

9. H. Zhu et al., Phys. Rev. Lett. 87, 081801-1 (2001).

10. S. Galster et al., Nucl. Phys. B 32, 221 (1971).

11. R. Schiavilla, I. Sick, Phys. Rev. C 64, 041002-1(R) (2001).

12. F. Cardarelli, A. Simula, Phys. Rev. C 62, 065201-1 (2000).

13. M.F. Gari, W. Krümpelmann, Z. Phys. A 322, 689 (1985).

14. M.F. Gari, W. Krümpelmann, Phys. Lett. B 274, 159 (1992).

15. G. Holzwarth, Z. Phys. A 356, 339 (1996).

16. S. Boffi et al., Nucl. Phys. A 699, 312c (2002).

17. R.F. Wagenbrunn, Phys. Lett. B 511, 33 (2001).

18. P. Mergell et al., Nucl. Phys. A 596, 367 (1996).

19. D. Merten et al., Eur. Phys. J. A 14, 477 (2002), hep$\mathrm{ph} / 0204024$

20. J.J. Kelly, Phys. Rev. C 66, 065203 (2002), hepph/0204239. 